immersed in an ultrasound washtub for 1 hour to ensure sloughing of the biofilm. Living bacteria were identified by plating on nutrient agar media and incubation for 48 hour at $37^{\circ} \mathrm{C}$.

Epifluorescent stains were used for the total bacteria count Lipopolysaccharide levels were determined by endotoxin activity measurements. Polyoside contents were determined by the colometric method, and the chemical oxygen demand was measured to evaluate the amount of organic substance. Biofilms detached from tubing samples drawn from the water path, bicarbonate path, and fresh dialysate path within dialysis machines contained approximately $1310^{3}-1310^{6}$ total bacteria/ $\mathrm{cm}^{2}$, yet only some living bacteria were found. Endotoxin levels ranged from 1 to $12 \mathrm{EU} / \mathrm{cm}^{2}$.

In contrast in the dialysate fluid, no bacteria were found, and the endotoxin content was below the detection level of the method. The polyoside content and chemical oxygen demand of the biomass ranged from 11 to $83 \mu \mathrm{g} / \mathrm{cm}^{2}$ and from 53 to $234 \mu \mathrm{g} / \mathrm{cm}^{2}$, respectively.

The authors concluded that a germ- and endotoxin-free dialysate does not exclude the risks and hazards of bacteria and endotoxin discharge from biofilm developed on the fluid pathway tubing, acting as a reservoir for continuous contamination, and efforts in the optimization of cleaning and disinfection procedures used for hemodialysis systems should aim to detach and neutralize biofilm when necessary.

FROM: Man NK, Degremont A, Darbord JC, Collet M, Vaillant P. Evidence of bacterial biofilm in tubing from hydraulic pathway of hemodialysis system. Artif Organs 1998;22:596-600.

\section{Microbial Contamination of Endoscopes After Manual Cleaning}

Colonoscopes and other types of endoscopes that are exposed to the intestinal tract present a special bacterial decontamination challenge because the colon has a large and diverse microbial population. Chu and coinvestigators from Advanced Sterilization Products, Irvine, California, conducted a study to determine the levels and types of microorganisms (bioburden) present on flexible gastrointestinal endoscopes. The bioburden of colonoscope insertion-tube surfaces and suction channels were determined after use and after manual cleaning. After use, the bioburden in suction channels averaged $7.0 \times 10^{9}$ colony-forming units (cfu); cleaning reduced this level to $1.3 \times 10^{5}$. Cleaning of tube surfaces reduced the after-use bioburden from a level of $5.1 \times 10^{5}$ to $2.2 \times 10^{4} \mathrm{cfu}$.

Gram-negative rods accounted for approximately $99 \%$ of the bioburden within the suction channel after use and after cleaning. After use, microbial flora were predominantly Escherichia coli and Bacteroides. The flora shifted to waterborne Pseudomonas organisms and other members of the family Enterobacteriaceae after cleaning. Gram-positive bacteria were the primary isolates from the device surfaces both after use (56\%) and after cleaning (47\%). Because gram-positive cocci and diphtheroids are a part of the normal microbiota of the skin, these bacteria may have been introduced by the hospital environment or by handling.

After the cleaning of in-use colonoscopes, fewer than $10^{6}$ vegetative bacteria could be recovered. This value is several logs lower than some previous estimates. This finding may be useful in the formulation of sterilization and disinfection cycles. Microflora from the colonoscopes indicated that the cleaning process introduced waterborne and enteric microorganisms, which highlights the importance of sanitation in the device reprocessing area.

FROM: Chu NS, McAlister D, Antonoplos PA. Natural bioburden levels detected on flexible gastrointestinal endoscopes after clinical use and manual cleaning. Gastrointest Endosc 1998;48:137-142.

\section{Acinetobacter baumanii Outbreak}

Acinetobacter baumanii is an important opportunistic pathogen that is evolving rapidly toward multidrug resistance and is involved in various nosocomial infections that often are severe. It is difficult to prevent $A$ baumanii infection because $A$ baumanii is ubiquitous and the epidemiology of the infections it causes is complex. Researchers at the Hotel-Dieu Hospital, Nantez, France, longitudinally analyzed the epidemiology of infections caused by $A$ baumanii in a medical and surgical intensive-care unit for almost 5 years and assessed the relation between fluoroquinolone use and the persistence of multidrug-resistant clones.

Three case-control studies and a retrospective cohort study were conducted in a 20-bed medical and surgical intensive-care unit. A baumanii was isolated from 45 patients in urine (31\%), the lower respiratory tract $(26.7 \%)$, wounds $(17.8 \%)$, blood $(11.1 \%)$, skin $(6.7 \%)$, cerebrospinal fluid (4.4\%), and sinus specimens (2.2\%). One death was due to A baumanii infection.

Antimicrobial resistance pattern and molecular typing were used to characterize isolates. The incidence of $A$ baumanii infection and the use of fluoroquinolones were calculated annually. Initially, 28 patients developed $A$ baumanii infection. Eleven isolates had the same antimicrobial susceptibility profile, genotypic profile, or both (epidemic cases), and 17 were heterogeneous (endemic cases). A surgical procedure done in an emergency operating room was the main risk factor for epidemic cases, whereas previous receipt of a fluoroquinolone was the only risk factor for endemic cases. The opening of a new operating room combined with the restriction of fluoroquinolone use contributed to a transitory reduction in the incidence of infection. When a third epidemiological study was done, previous receipt of a fluoroquinolone was again an independent risk factor, and a parallel was seen between the amount of intravenous fluoroquinolones prescribed and the incidence of endemic infection. Epidemic infections coexisted with endemic infections favored by the selection pressure of intravenous fluoroquinolones.

FROM: Villers D, Espaze E, Coste-Burel M, Giauffret F, Ninin E, Nicolas F, et al. Nosocomial Acinetobacter baumanii infections: microbiological and clinical epidemiology. Ann Intern Med 1998;129:182-189.

Additional news items in this issue: Global Problem of DrugResistant Mycobacterium tuberculosis, page 753; Multidrug-Resistant Pneumococcal Pneumonia in a Nursing Home, page 777; VRE in Chronic Hemodialysis Patients, page 794. 